\title{
Distribuição arterial na parede gástrica de gatos
}

\author{
Arterial distribution in the gastric wall of cats
}

\author{
Caroline Medeiros Bastos (D), Tathiana Alves Marques (D), Thais Alves Fernandes (D), Marcelo Abidu-Figueiredo (D), \\ Paulo Roberto Bernardes Lopes (D), Marcelo Salvador Gomes (D), Luciano da Silva Alonso (D)* \\ Universidade Federal Rural do Rio de Janeiro (UFRRJ), Seropédica, RJ, Brasil
}

\section{Resumo}

As origens e ramificações das artérias que irrigam o estômago de animais domésticos estãobem determinadas na literatura, mas a forma como se dá a distribuição destes vasos na parede do estômago ainda não foi completamente estabelecida. 0 objetivo desta pesquisa foi descrever a distribuição arterial macroscópica na superfície do estômago de gatos. As dissecções foram realizadas em 30 cadáveres de gatos adultos, 15 machos e 15 fêmeas, com média do comprimento rostrossacral de 47,9 cm e 46,6 cm, respectivamente. Os gatos foram posicionados em decúbito lateral direito e foi realizada

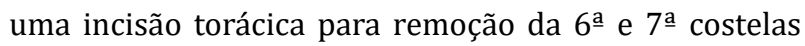
para canulação da porção torácica da aorta. Em seguida, o sistema vascular foi lavado com solução salina, fixado com solução de formaldeído a 10\% e preenchidos com solução de Petrolátex S-65 corado. Após cinco dias imersos em solução de formaldeído a $10 \%$, todos os animais foram lavados em água corrente e dissecados. Foi utilizado o modelo de divisão do estômago em quadrantes cárdicos e pilóricos. Para a comparação do número de ramos entre os quadrantes, utilizou-se o teste de ANOVA com pós-teste de Tukey; e para comparação dos valores obtidos em cada quadrante nos dois sexos foi utilizado o teste T não pareado. Toda a análise estatística foi feita no software Grafpad Prism 5, considerando p < 0,05 como significativo. Com dissecação e quantificação dos vasos na superfície gástrica, observou-se que houve diferença na distribuição arterial entre os quadrantes tanto em machos quanto em fêmeas, e que a face visceral é a mais vascularizada (média de 75,20 vasos em machos e de 76,39 vasos em fêmeas). Quanto aos quadrantes, a maior concentração de vasos se deu nos quadrantes pilóricos, tanto em machos (77,19 vasos) como em fêmeas (83,66 vasos). Nos quadrantes cárdicos foram contabilizados 30,47 vasos em média nos machos e 43,39 vasos em fêmeas. Houve diferença na distribuição arterial entre os diferentes quadrantes ( $p<0,0001)$ em ambos os sexos.

Palavras-chave: Gato. Estômago. Vascularização. 


\section{Abstract}

The origins and ramifications of arteries that irrigate the stomach of domestic animals are well established in the literature; however, the pattern of the distribution of these vessels in the wall of the stomach of domestic animals has not yet been fully established. The aim of this research was to describe the macroscopic arterial distribution on the stomach surface of cats. Anatomical dissections were performed on 30 cadavers of adult cats, 15 male and 15 female, with a rostrum-sacral length of $47.9 \mathrm{~cm}$ and $46.6 \mathrm{~cm}$, respectively. Cats were positioned in right lateral decubit and a thoracic incision was made for removing the 6th and 7 th ribs to cannulate the thoracic portion of the aorta. The vascular system was washed with saline solution, fixated with 10\% formaldehyde solution, and then filled with colored Petrolattex S-65. After five days in 10\% formaldehyde solution, all the animals were washed in current water and dissected. The model of division of the stomach was used in cardiac and pyloric quadrants. For the comparison of the number of branches between the quadrants, ANOVA test with Tukey post-test was used, and for the comparison of the values obtained in each quadrant in both sexes, unpaired T test was used. Grafpad Prism 5 software was used, considering $p<0.05$ as statistically significant. With the dissection and quantification of the vessels on the gastric surface, we observed a difference in the arterial distribution between the quadrants in both males and females. We observed that the visceral face was the most vascularized (mean of 75.20 vases in males and 76.39 vases in females). Regarding the quadrants, the highest concentration of vessels occurred in the pyloric quadrants, both in males (77.19 vessels) and in females (83.66 vessels). In the cardiac quadrants, 30.47 vessels were recorded on average in males and 43.39 in females. A difference was noted in the arterial distribution between the different quadrants $(p<0.0001)$ in both males and females.

Keywords: Cat. Stomach. Vascularization.

\section{Introdução}

O estômago é um órgão em forma de saco, localizado na região hipocondríaca esquerda do abdômen. A sua estrutura, macro e microscópica, cumpre as funções de reservatório alimentar temporário e de digestão química inicial dos alimentos (Ellenport, 1986).

Anatomicamente, possui duas faces: uma parietal, relacionada com o fígado, parte esquerda do diafragma e parede abdominal esquerda e ventral; e uma visceral, menos extensa e mais achatada do que a outra, direcionada dorsal e medialmente e justaposta aos intestinos, pâncreas e rim esquerdo. Apresenta duas curvaturas, sendo que a maior está encostada na parede abdominal esquerda e tem grande capacidade de distensão, e a menor, e consideravelmente mais reduzida, forma na sua parte inferior um ângulo agudo, a incisura angular. Delimitam-se ainda duas extremidades, a esquerda ou fundo, que é volumosa e arredondada, e corresponde a parte mais dorsal do órgão, e a direita ou pilórica, direcionada cranial e dorsalmente (Duarte, 2009).

No gato, o estômago possui ângulos mais fechados, dobrando-se sobre si mesmos, e o seu piloro não atinge normalmente o antímero direito do abdômen. A capacidade de dilatação também não se compara a do estômago dos canídeos, uma vez que os seus hábitos alimentares são muito diferentes, proporcionando ao longo do dia pequenas e frequentes refeições (Dyce et al., 2010).

0 estômago pode ser dividido em cinco regiões: cárdia, fundo, corpo, antro e piloro. Funcionalmente, porém, consideram-se duas dessas regiões, que são o corpo (proximal) e o antro (distal). 0 corpo é capaz de acomodar grande quantidade de alimento em um determinado espaço de tempo e tem como função produzir secreções mucosas, ácido clorídrico e proteases através das glândulas gástricas; o antro está mais distal, e sua função é produzir solução alcalina e pobre em enzimas através de glândulas cardíacas e pilóricas (Dyce et al., 2010).

Analisando a histologia de animais em geral, Zahariev et al. (2010) relatam que a maioria dos órgãos tubulares, como o estômago, é formada por quatro camadas concêntricas: túnica serosa, muscular, submucosa e mucosa.

A mucosa gástrica nos gatos e cães é do tipo glandular, com glândulas cárdicas, fúndicas e pilóricas. Em gatos, entretanto, há uma camada compacta subglandular na mucosa do estômago, ausente em cães (Zahariev et al., 2010). 
A vascularização do estômago de animais de diferentes espécies já foi objeto de estudo em diversas pesquisas, pois o conhecimento da distribuição arterial na parede deste órgão assume grande importância em experimentos que avaliam as funções gástricas e sistêmicas em função das variações na anatomia do estômago e de seus vasos (Xavier-Silva et al., 2012; Oliveira et al., 2017).

Vasos responsáveis pela irrigação gástrica se originam da artéria celíaca, primeiro ramo da aorta abdominal, localizada após o hiato aórtico, na cavidade abdominal. A artéria celíaca está entre os principais vasos sanguíneos que partem do segmento abdominal da aorta, pois irriga vísceras importantes como fígado, estômago, baço, pâncreas, além de parte do intestino delgado (Siston, 2011). A irrigação do estômago tem origem neste vaso, que emite as artérias lienal, gástrica esquerda (AGE) e hepática, as quais contribuem para a irrigação sanguínea do órgão.

Xavier-Silva et al. (2011) descreveram as principais ramificações da artéria lienal em gatos em estudo onde verificou-se que a artéria lienal se originou diretamente da artéria celíaca e emitiu ramos para o baço, curvatura maior do estômago e lobo esquerdo do pâncreas. A artéria lienal emite as artérias gástricas curtas para a grande curvatura do estômago e bifurca-se em uma artéria gastroepiplóica esquerda e uma artéria omental, que margeia a curvatura maior. Estas últimas também contribuem para a vascularização. A AGE chega ao órgão junto à curvatura menor, na região de inserção do mesogástrio, e emite ramos para as superfícies visceral e parietal do estômago (Dyce et al., 2010). As veias gástricas drenam na veia porta-hepática. Apesar de bem conhecidas, as origens destes vasos em gatos e outros animais domésticos, e informações quantitativas a respeito do comportamento dos mesmos ao atingirem a superfície do estômago ainda são pouco estudadas.

Em trabalho realizado com equinos, Alonso (2005) descreveu aspectos da vascularização do estômago, com ênfase nos principais vasos que atingem a superfície do órgão; o autor fez maior menção ao comportamento da AGE, estando os demais vasos com descrição insuficiente para fins de inferência acerca de maior ou menor capacidade de perfusão sanguínea na mucosa gástrica. 0 autor correlacionou achados macroscópicos, obtidos através da divisão do órgão em quadrantes, com o intuito de definir um parâmetro de fácil compreensão, com aspectos microscópicos de elementos da vascularização sobrepostos em lâminas histológicas da mucosa glandular.

Xavier-Silva et al. (2012) observaram que a artéria gástrica esquerda pode se originar diretamente da artéria celíaca ou ter sua origem através da formação de um tronco comum com a artéria lienal.

Este trabalho teve como objetivo descrever aspectos macroscópicos quantitativos da distribuição arterial na parede do estômago de gatos.

\section{Material e métodos}

Este experimento faz parte do projeto intitulado Anatomia vascular no gato: estudo destinado à prática cirúrgica, experimental e ao diagnóstico por imagem, que foi submetido e aprovado pela Comissão de Ética e Pesquisa da Universidade Federal Rural do Rio de Janeiro, sob o processo $\mathrm{n}^{\circ}$ 23083.005334/2009-49. Foram utilizados cadáveres de 30 gatos, SRD, adultos, sendo 15 machos e 15 fêmeas, os quais foram cedidos à área de Anatomia Animal da Universidade Federal Rural do Rio de Janeiro por clínicas veterinárias e pelo Hospital Veterinário de Pequenos Animais da UFRRJ, cujos óbitos não estavam relacionados aos objetivos deste estudo. Estavam congelados e foram armazenados em freezer desde que foram recebidos. Após o descongelamento, os cadáveres foram posicionados em decúbito lateral direito para acesso à porção torácica da aorta, através de ressecção dos músculos intercostais e costelas, realizada entre o $6^{\circ}$ e o $10^{\circ}$ espaço intercostal. A artéria foi canulada com sonda uretral número oito ou dez (variável em função do diâmetro do vaso), sendo presa com barbante para evitar extravasamentos e manter a pressão intravascular. A fixação foi feita injetando-se, através da sonda, solução de formaldeído a $10 \%$ (volume variável de 40 a $80 \mathrm{ml}$ em função do peso do cadáver) em sentido caudal. 
Imediatamente após a fixação dos cadáveres, injetou-se solução de Petrolátex S65 (Refinaria Duque de Caxias - REDUC- Petrobrás, Duque de Caxias, RJ) corado com pigmento Suvinil de cor vermelha para preenchimento de todo o sistema arterial. Em seguida, os cadáveres foram acondicionados em caixas de polietileno de baixa densidade com capacidade de 500 litros, contendo solução de formaldeído a 10 \% para a finalização do processo de fixação e coagulação do látex. Decorridos sete dias da injeção do látex, os cadáveres foram lavados em água corrente e posteriormente dissecados com o intuito de localizar as ramificações da artéria celíaca e avaliar quantos ramos cada uma destas emitem para irrigação da parede gástrica. Após o estudo, os cadáveres foram disponibilizados para finalidades de ensino.

A análise macroscópica quantitativa dos vasos foi realizada a partir da divisão do estômago em quadrantes, seguindo a metodologia adotada por Alonso et al. (2008). Para isto, dois eixos imaginários, perpendiculares entre si, foram posicionados nos sentidos longitudinal e transversal do estômago (Figura 1). 0 eixo $\mathrm{Y}$ foi ordenado no sentido longitudinal, entre a cárdia e o piloro, que correspondem, respectivamente, o esfíncter entre o esôfago e estômago e o esfíncter entre o estômago e o duodeno. $\mathrm{O}$ eixo $\mathrm{X}$ foi posicionado transversalmente, passando pelo ponto médio entre a cárdia e a incisura angular, que é uma reentrância existente entre a cárdia e o piloro. Desta forma, constituíram-se os quadrantes denominados cárdico parietal (QCP), cárdico visceral (QCV), pilórico parietal (QPP) e pilórico visceral (QPV), apresentados em plano único, com vista superficial externa.

0 número de ramificações da artéria gástrica esquerda e direita, artéria lienal, artérias gástricas curtas e artérias gastroepiplóicas esquerda e direita nos quadrantes QCP, QCV, QPP e QPV do estômago (Figuras 2, 3 e 4) foram expressos em média e desvio padrão. Para a comparação do número de ramos entre os quadrantes, utilizou-se o teste de ANOVA com pós-teste de Tukey. Para comparação dos valores obtidos em cada quadrante nos dois sexos foi utilizado o teste T não pareado. Utilizouse software Grafpad Prism 5, considerando $\mathrm{p}<0,05$ como significativo.

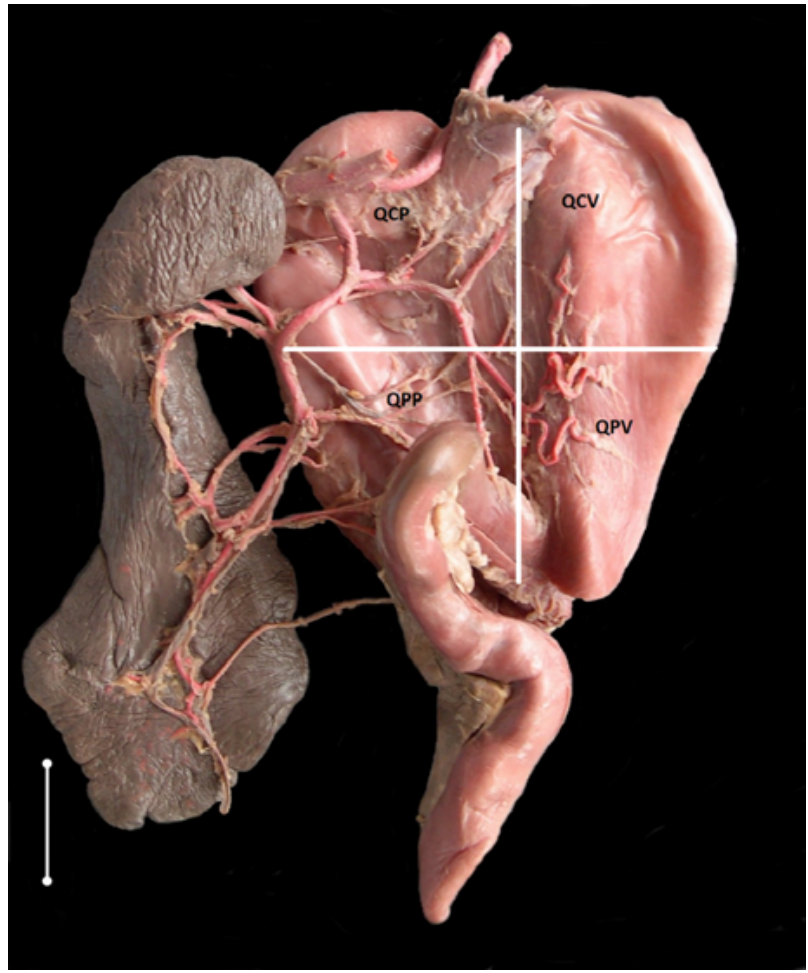

Figura 1 - Fotomacrografia do estômago e baço de gato (fêmea), com incisão ao longo da curvatura maior do estômago e em vista dorsal. $\mathrm{Q} C P=$ quadrante cárdico parietal $; \mathrm{Q} C V$ = quadrante cárdico visceral; $\mathrm{QPP}=$ quadrante pilórco parietal; $\mathrm{QPV}=$ quadrante pilórico visceral. Ramificações arteriais para a parede gástrica dissecadas e injetadas com látex vermelho. Seropédica, RJ, 2015. Barra de escala: $1 \mathrm{~cm}$.

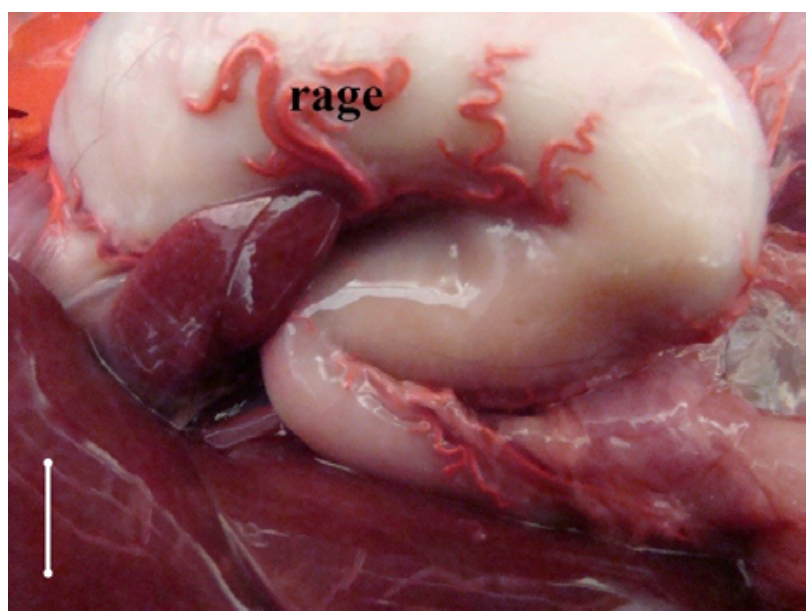

Figura 2 - Fotomacrografia da face visceral do estômago de gato. Técnica: injeção de látex corado na artéria gástrica esquerda. rage: ramificações da artéria gástrica esquerda. Seropédica, RJ, 2015. Barra de escala: $1 \mathrm{~cm}$. 


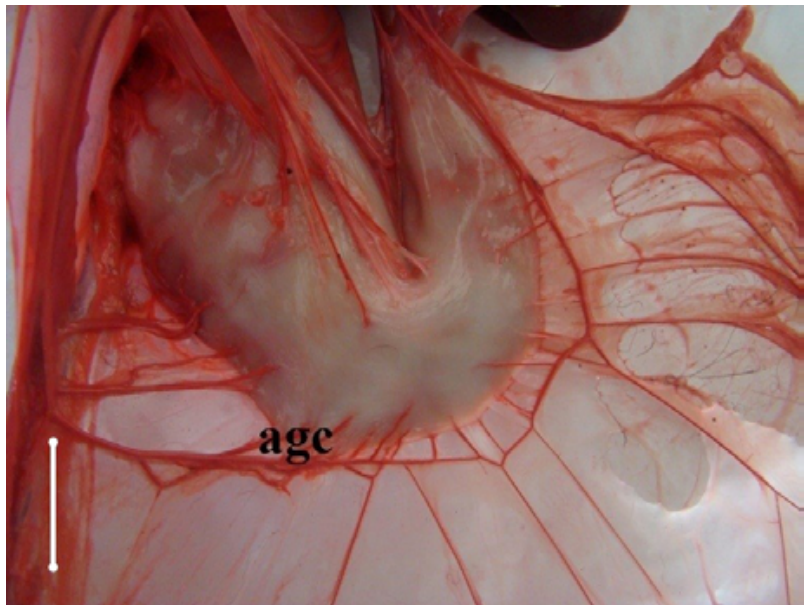

Figura 3 - Fotomacrografia da curvatura maior do estômago de gato. Técnica: injeção de látex corado nas ramificações da artéria celíaca. agc: ramificações da artérias gastroepiplóicas (artérias gástricas curtas). Seropédica, RJ, 2015. Barra de escala: $1 \mathrm{~cm}$.

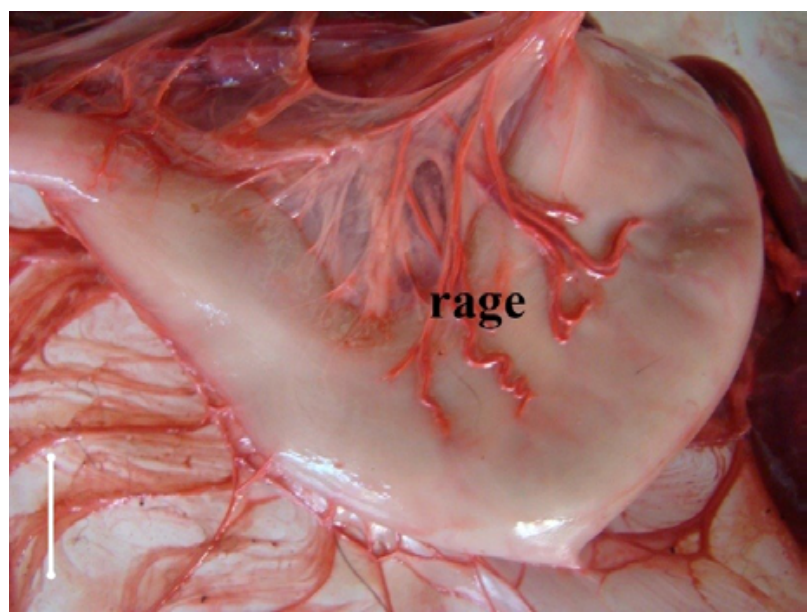

Figura 4 - Fotomacrografia da face parietal do estômago de gato. Técnica: injeção de látex corado nas ramificações da artéria celíaca. rage: ramificações da artéria gástrica esquerda. Seropédica, RJ, 2015. Barra de escala: $1 \mathrm{~cm}$.

\section{Resultados}

A artéria celíaca emergiu da região ventral da porção abdominal da aorta, próximo ao hiato aórtico do diafragma; apresentou-se como um vaso único. Emitiu as artérias gástrica esquerda, hepática e lienal. A artéria gástrica esquerda se insere na curvatura menor, próximo ao esfíncter cárdia e irriga ambas as superfícies do estômago.
Observou-se que a face visceral é a mais vascularizada (Figura 1), com média de 75,20 vasos em machos e de 76,39 vasos em fêmeas. Quanto aos quadrantes, a maior concentração de vasos se deu nos quadrantes pilóricos, tanto em machos (77,19 vasos) como em fêmeas (83,66 vasos). Nos quadrantes cárdicos foram contabilizados 30,47 vasos em média nos machos e 43,39 vasos em fêmeas. Há diferença na distribuição arterial entre os diferentes quadrantes ( $p<0,0001)$ tem ambos os sexos.

Não há diferença estatística no padrão de distribuição arterial por quadrantes comparando machos e fêmeas $(p>0,05)$.

\section{Discussão}

No presente estudo, a artéria celíaca se apresentou como um vaso ímpar se originando diretamente da aorta. As vezes a artéria celíaca se origina com a artéria mesentérica cranial por um tronco comum denominado tronco celíaco-mesentérico. Esta variação anatômica foi caracterizada em cães (Schmidti e Schoenau 2007), gatos (Roza et al., 2009) e gambá (Didelphis albiventris) ( Culau et al., 2010).

Neste estudo foi realizada a quantificação dos ramos terminais das artérias que suprem o estômago, sendo considerados como ramos terminais todos os que foram identificados penetrando diretamente na parede gástrica. Em trabalhos anteriores realizados em estômagos de equinos (Alonso et al., 2008) e de coelhos (Siston et al., 2015), os autores contabilizaram também ramificações de segunda e terceira ordem, considerando a artéria gástrica esquerda como vaso de primeira ordem, na sequência de ramificações a partir da artéria celíaca. Entretanto quantificar a ramificação diretamente na parede gástrica, como foi realizado no presente trabalho, pode ser mais prático e não acarreta prejuízo para a avaliação da distribuição arterial nos quadrantes da superfície externa do estômago.

No trabalho realizado com equinos, Alonso et al. (2008) observaram que a superfície externa dos quadrantes pilóricos mostrou-se com mais ramificações da AGE em comparação aos quadrantes 
cárdicos. Em coelhos, Siston et al. (2015) observaram que o quadrante pilórico visceral de fêmeas é mais vascularizado do que em estômago de machos e, no geral, os quadrantes pilóricos também apresentaram maior presença de ramos da AGE do que os quadrantes cárdicos. Neste trabalho não foram considerados aspectos biológicos do tipo de alimentação e nem relações filogenéticas. Tais parâmetros são importantes em estudos de anatomia comparada, mas o aspecto central deste trabalho foi a descrição do comportamento arterial na parede gástrica de gatos e a avaliação quantitativa para contribuir em estudos posteriores de anatomia comparada. Em relação à técnica, tanto no trabalho realizado em equinos (Alonso et al., 2008) quanto no realizado em coelhos (Siston et al., 2015) foram contabilizadas ramificações de segunda e terceira ordem a partir da artéria celíaca, da artéria lienal e da artéria hepática.

Neste estudo com gatos, observou-se que a quantificação de vasos diretamente no momento em que penetram na parede gástrica é suficiente para estabelecer padrões de vascularização nos quadrantes, não sendo necessário contabilizar as ramificações secundárias e terciárias, exatamente porque tais níveis de ramificações não tocam a parede gástrica. 0 que se observa neste trabalho é que quantificar ramos de artérias gástricas diretamente na parede do órgão confere maior agilidade, sem prejuízo nos resultados e na acurácia do método.

\section{Conclusão}

Nas condições do presente estudo, observouse diferença na distribuição arterial na parede gástrica de gatos entre os diferentes quadrantes, tanto em machos quanto em fêmeas. Em ambos os sexos, o quadrante com maior número de irrigações é o quadrante pilórico visceral, seguido do cárdico visceral e do pilórico parietal, e o menos vascularizado é o cárdico parietal. A artéria que emite o maior número de ramos para a superfície estomacal é a artéria gástrica esquerda.

\section{Referências}

Alonso LS. Aspectos macroscópicos e microscópicos da vascularização sangüínea na parede do estômago de equinos [tese]. São Paulo: Universidade de São Paulo; 2005. 101 p.

Alonso LS, Resende HRA, Duboc MV, Silva LCLP. Distribuição arterial na superfície do estômago de equinos e relação com a área superficial do órgão. Braz J Vet Res Anim Sci. 2008;45(4):320-6.

Culau POV, Reckziegel SH, Goltz LV, Araújo ACP. A artéria celíaca em Didelphis albiventris (gambá). Acta Sci Vet. 2010;38(2):121-5.

Dyce KM, Sack WO, Wensing CJG. Tratado de Anatomia Veterinária. 4 ed. Rio de Janeiro: Elsevier; 2010. 834 p.

Duarte ARR. Pesquisa de Helicobacter spp. em felinos [dissertação]. Lisboa: Universidade Técnica de Lisboa; 2009. $148 \mathrm{p}$.

Ellenport CR. Sistema Digestivo. In: Getty R. Anatomia dos animais domésticos. Rio de Janeiro: Guanabara Koogan; 1986. p. 1445-64.

Oliveira GB, Oliveira REM, Bezerra FVF, Câmara FV, Araújo Jr HN, Oliveira MF. Origem e distribuição da artéria celíaca em preás (Galea spixii Wagler, 1831). Cienc Anim Bras. 2017;18:e-32918.

Roza MS, Pestana FM, Hernandez JMF, Silva BX, AbiduFigueiredo M. Tronco Celiaco-Mesentérico em Gato. Rev Port Cienc Vet. 2009;104(569-572):83-6.

Schmidt DP, Schoenau LSF. Origem das artérias celíaca e mesentérica cranial por tronco comum em cão. Cienc Rural. 2007;37(2):408-11.

Siston NM. Estudo macroscópico da vascularização do estômago de coelhos Nova Zelândia [monografia]. Seropédica: Universidade Federal Rural do Rio de Janeiro; 2011. 38 p. 
Siston NM, Sousa CAS, Gomes MS, Alonso LS, AbiduFigueiredo M. Estudo macroscópico da vascularização do estômago de coelhos (Oryctolagus cuniculus) Nova Zelândia: divisão em quadrantes. Rev Acad Cienc Anim. 2015;13:31-7.

Xavier-Silva B, Marinho GC, Alonso LS, AbiduFigueiredo M. Ramificações principais da artéria gástrica esquerda no gato doméstico. Rev Port Cienc Vet. 2012;111(581-582):51-6.
Xavier-Silva B, Roza MS, Hernandez JMF, Souza HJM, Abidu-Figueiredo M. Artéria lienal em gatos: estudo aplicado à pesquisa anatômica e a prática cirúrgica. Rev Bras Med Vet. 2011;33(1):41-7.

Zahariev P, Sapundzhiev E, Pupaki D, Rashev P, Palov A, Todorov T. Morphological characteristics of the canine and feline stomach mucosa. Anat Histol Embryol. 2010;39(6):563-8. 